\title{
A Fourier series based Template Matching Approach to Detect the Splitting of Second Heart Sound
}

\author{
Anandarup Mukherjee ${ }^{\mathrm{a}}$, Angshuman Khan ${ }^{\mathrm{b}}$ \\ ${ }^{a, b}$ (Dept. of Electronics \& Communication Engg., University of Engineering \& Management, Jaipur, India)
}

\begin{abstract}
In this paper, we present a method of detecting the splitting of heart sound S2 based on a template matching approach. A mathematical model of normal $S 2$ is created and tested on other normal 52 complexes for goodness of fit. The generalized mathematical model is used as a template for detecting splitting of S2 sounds, as they will not generate a good fit with a model of a normal $S 2$ complex. This automated detection of splitting of $S 2$ can be used in the detection of congenital heart diseases or pulmonary hypertension. We have provided an estimate of the fit results of the mathematically synthesized $S 2$ complex with the split $S 2$ complexes.
\end{abstract}

Keywords: Curve fitting, Fourier Series, Goodness of fit, Phonocardiography, Splitting of S2.

\section{Phonocardiography}

The diagnostic procedure of generating a graphical registration of the sounds and murmurs produced in the heart, its valves and great vessels is called phonocardiography. The graphical record of phonocardiogram is called phonocardiograph (PCG) [1]. A typical phonocardiograph is recorded using microphones, which are kept on the human thorax; the procedure is quite similar to using a stethoscope. Sounds in the frequency range of $25 \mathrm{~Hz}$ to $100 \mathrm{~Hz}$ are filtered out by the microphones. The fundamental advantages of PCG are:

a. The sounds which are below of human hearing range can be recorded;

b. Sounds can be exactly timed with the cardiac cycle;

c. The diagnostically significant properties like pitch, intensity or duration of sounds can also be analyzed.

\subsection{Heart sounds}

The generation of heart sounds can be attributed to some special mechanical events within the heart, such as those caused by the closing \& opening of the heart valves or the sudden distension of the arterial walls at the time of ejection or during contraction of the myocardium. Amongst the reasons for generation of heart sounds, the closure sound of valves is most significant as they are responsible for the generation of high frequency sounds. The first heart sound is called S1 or systolic sound or 'lub'. It is a high frequency sound and is related to opening and closing of Mitral \& Tricuspid valve. This sound appears 0.02 seconds to 0.04 seconds after the occurrence of QRS complex. It lasts for duration of 0.12 seconds to 0.15 seconds. The first heart sound is attributed to the closing of the tricuspid \& mitral valves. This sound is best heard in the area of mitral and tricuspid auscultator. This sound precedes the sound of carotid pulse upstroke \& it synchronous with the apex beat. The intensity of S1 may vary depending on the atrio-ventricular valve mobility. S1 has four components and when compared to an electrocardiogram (ECG), the first component starts at the peak of the R wave of the QRS complex.

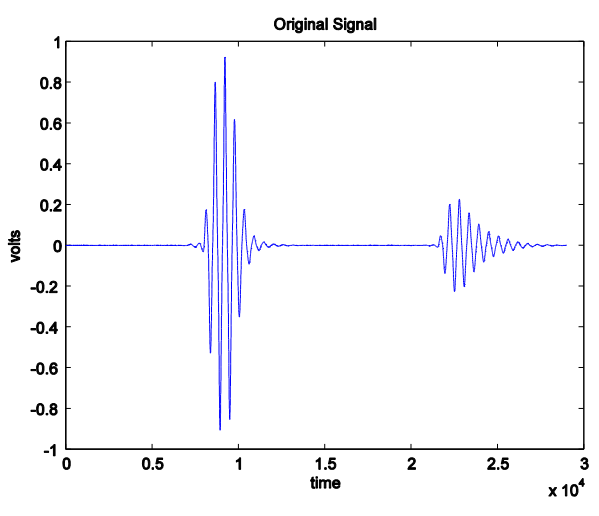

Figure 1: A normal PCG reading of S1-S2 complex

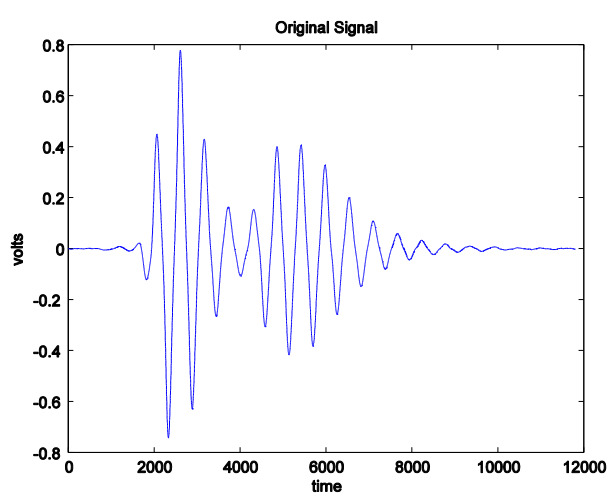

Figure 2: A PCG recording showing splitting of S2 
The second heart sound is known as S2 or diastolic sound or 'dub'. It is also a high frequency sound \& related to closing and opening of the semi-lunar valves. In the terminal period of the $\mathrm{T}$ wave, it appears \& lasts for 0.08 seconds to 0.12 seconds within a frequency range $50 \mathrm{~Hz}$ to $70 \mathrm{~Hz}$. This sound mainly arises due to the closure of aortic valve and pulmonic valve. S2 starts with a gap of $5 \mathrm{~ms}$ after closure of the aortic valves \& synchronous with dicrotic notch. S2 is best heard at the second left inter-space, near the sternal border. The second heart sound, S2, also consists of four components, i.e. S2A, S2B, S2C \& S2D. S2A is a low pitched vibration. It is due to the ventricular relaxation during a commencing diastole. The S2B represents the closure sound of the semi-lunar valves $\&$ has high amplitude as well as high frequency. The third component represents blood passing through arteries and is composed of one or two coarse vibrations of smaller amplitudes. It is vascular in origin. The S2D component is due to the opening of the mitral and tricuspid valve with a low vibration. Figure-1 illustrates a normal S1 and S2 heart sound from a PCG [10], [11].

\subsection{Splitting of the Second Heart Sound:}

Splitting of the second heart sound is generally found in the pulmonary area of healthy children. The existence of aortic and pulmonary vessels is the indication of second sound splitting. In the case of congenital heart disease, splitting of second sound is an important factor. If S2 splitting is found, it signifies the occurrence of S2A due to closure of the aortic valves and occurrence of S2B due to the closure of pulmonary valves. In congenital heart disease or pulmonary hypertension accentuation, the diagnosis of other S2 components is important [10]. In the aortic valve and apical valve areas S2 is generally single. Pathologically S2 splitting can be of three types:

i. $\quad$ Fixed splitting: Due to the atrial septal defect (ASD)

ii. Large variable splitting(A2P2): Due to the late systole of right ventricle

iii. Paradoxial splitting (P2A2): Due to late systole of left ventricular \& early closure of pulmonary valve.

Figure 2 illustrates the PCG recording of the splitting of second heart sound.

\section{Curve Fitting}

The main objective of curve fitting is to generate a function or a curve such that it satisfies the given set of data points. The main task associated with curve fitting is the determination of coefficients and other parameters that fit best in the curve. It helps in generating a pattern or trend that is generally invisible in normal data points. The advantage of curve fitting is that it sets the seemingly random points into an arranged format making it more informative and in some cases even eliminating the noise and unwanted data. There are many types of curve fitting methods that are used, depending on the desired result and the available data like the Linear curve fitting, Polynomial curve fitting and Regression. Curve fitting can be further classified or extended to Interpolation and Smoothing. Interpolation is the process of obtaining a data or point anywhere along the generated curve that fits exactly to the given data. When this curve is used to find the points beyond the given set of data it is called Extrapolation i.e., estimating the next possible data. Smoothing involves generating a curve that is not an exact but an approximate fit to the data. The diverse usage of curve fitting has gained the interest of many researchers and over the years there have been many approaches to curve fitting, making it more efficient and robust [4], [5]. M. Gulsen et al. [6] gave a genetic algorithm based approach to curve fitting with multiple parameters and coefficients. Liang and David [7] proposed a method of fitting multi parametric curve to disordered and erroneous data by using non linear minimization. Due to its ability of visualizing and decreasing the randomness of the data, curve fitting finds its use in statistical analysis, biological signal modeling and establishing relationship or connection between the biological events that follow such as ECG Restitution Curve Analysis, Metabonomic Toxicology [8], modeling the kinetic flow of Bolus fluids [9]

\subsection{Database}

\section{Methodology}

The database for this paper is taken from the University of Michigan, Heart Sound and Murmur library. This database contains the PCG recordings of various types of heart sounds and murmurs, recorded and encoded in '.mp3' format with a bit-rate of $128 \mathrm{kbps}$ [12]. The recordings are primarily divided into three parts, based on the place of recording of data from the human body, viz. Apex area, aortic area and pulmonary area. In our work, we have taken into account, the recordings from the apex area only. Our selection from the database reflects a normal S1-S2 database and three types of murmurs, namely, early systolic, mid- systolic and holosystolic murmur. 


\subsection{Flowchart}

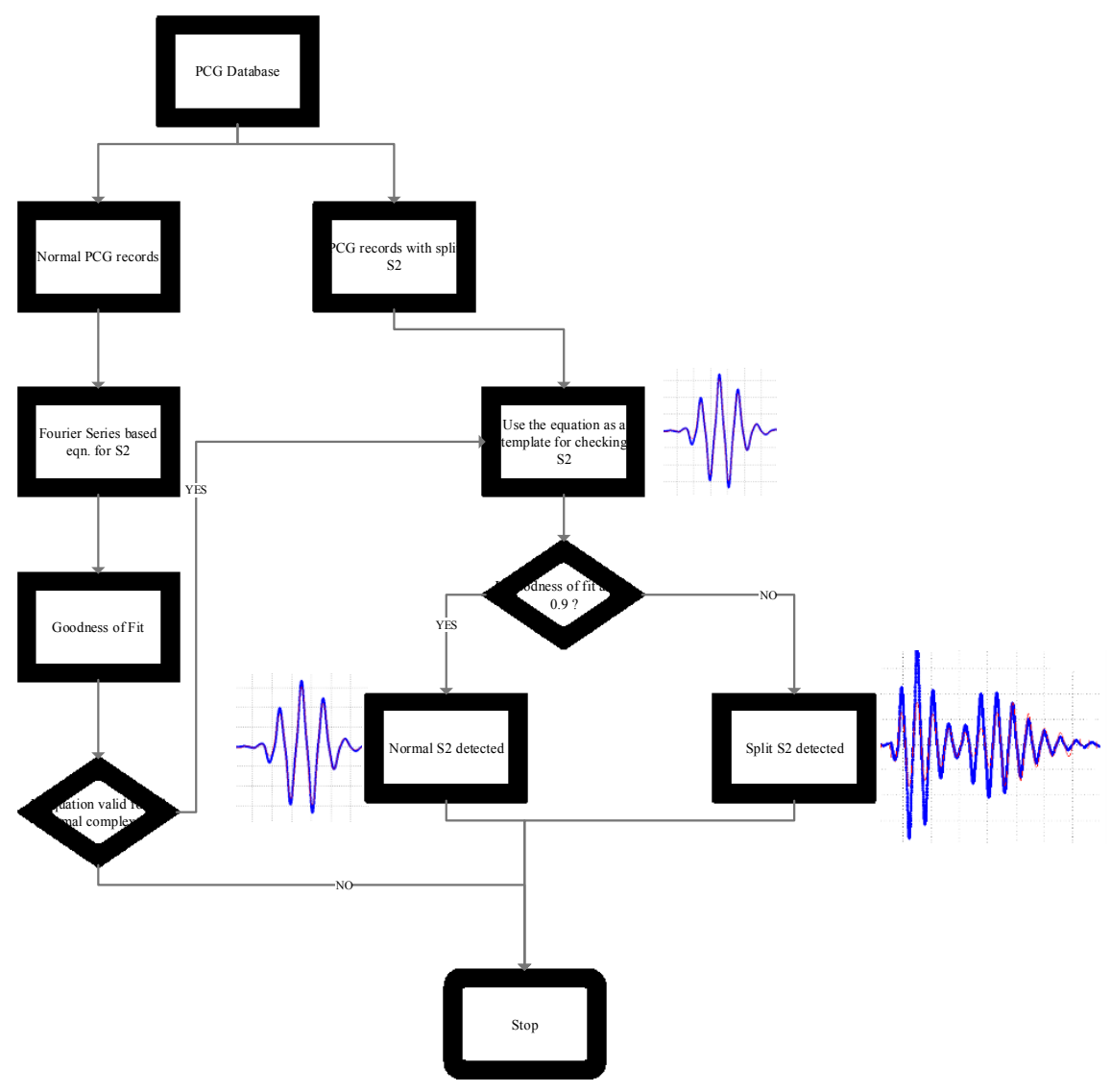

Figure 1: A flowchart describing the methodology followed in this paper.

\section{Results}

\subsection{TABLE OF FOURIER COEFFICIENTS}

The table of coefficients (table-1) lists the mean coefficients determined by the $8^{\text {th }}$ order Fourier series decomposition of normal S2 complexes. This synthesized curve for normal S2 gives an average goodness of fit, determined by $R^{2}$ value, of approximately 0.9818 for all normal $\mathrm{S} 2$ complexes. The values listed in table- 1 are used for formulating equation 1 .

Table 1: A table of the mean $8^{\text {th }}$ Order Fourier Coefficients for synthesizing a normal S2 complex

\begin{tabular}{|c|c|c|c|c|c|c|c|c|}
\hline $\mathbf{a}_{\mathbf{0}}$ & $\mathbf{a}_{\mathbf{1}}$ & $\mathbf{a}_{\mathbf{2}}$ & $\mathbf{a}_{\mathbf{3}}$ & $\mathbf{a}_{\mathbf{4}}$ & $\mathbf{a}_{\mathbf{5}}$ & $\mathbf{a}_{\mathbf{6}}$ & $\mathbf{a}_{7}$ & $\mathbf{a}_{\mathbf{8}}$ \\
\hline-0.001178 & -0.000335 & -0.001958 & 0.006822 & 0.001567 & 0.0101614 & -0.07982 & 0.1046 & -0.0655 \\
\hline $\mathbf{b}_{\mathbf{1}}$ & $\mathbf{b}_{\mathbf{2}}$ & $\mathbf{b}_{\mathbf{3}}$ & $\mathbf{b}_{\mathbf{4}}$ & $\mathbf{b}_{\mathbf{5}}$ & $\mathbf{b}_{\mathbf{6}}$ & $\mathbf{b}_{7}$ & $\mathbf{b}_{\mathbf{8}}$ & $\boldsymbol{\omega}$ \\
\hline-0.000572 & 0.004044 & -0.00969 & -0.01401 & 0.09801 & -0.195 & 0.1789 & -0.0647 & 0.001472 \\
\hline
\end{tabular}

\subsection{EQUATION OF SYNTHESIZED CURVE}

The equation in (1) is used as the template for distinguishing normal S2 sounds from Split S2 sounds in PCG recordings of various subjects. The template for matching, generated by the equation in (1) is illustrated in figure 4.

$$
\begin{aligned}
f(x)= & -0.001178-0.000335 \cos (x \omega)-0.001958 \cos (2 x \omega)+0.006822 \cos (3 x \omega)+0.001567 \cos (4 x \omega) \\
& +0.01614 \cos (5 x \omega)-0.07982 \cos (6 x \omega)+0.1046 \cos (7 x \omega)-0.0655 \cos (8 x \omega)-0.000572 \sin (x \omega) \\
& +0.004044 \sin (2 x \omega)-0.00969 \sin (3 x \omega)-0.0140 \sin (4 x \omega)+0.0980 \sin (5 x \omega)-0.195 \sin (6 x \omega) \\
& +0.1789 \sin (7 x \omega)-0.0647 \sin (8 x \omega)
\end{aligned}
$$




\subsection{RESULT OF TEMPLATE BASED DETECTION FOR S2 SPLITTING}

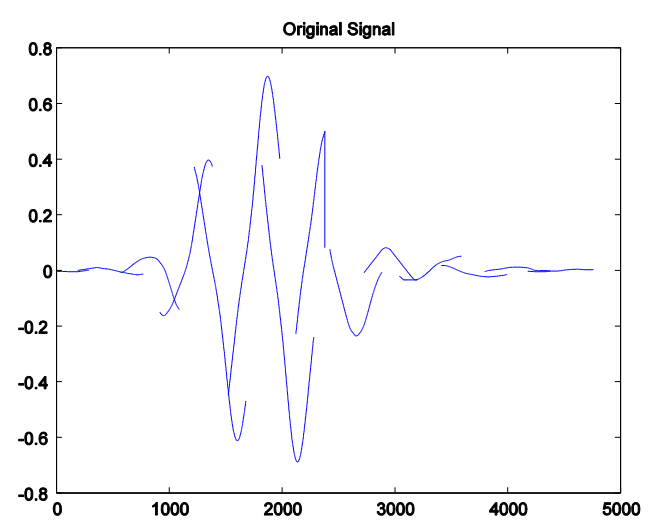

Figure 2: A plot of the synthesizing equation given in (1), which acts as the template for detection of Split S2

The following figures, from fig. 5 to 7 depict the use of the synthesized template for matching to the original signal. It is evident in figure 5, that the synthesized signal gives a good fit when tested with a normal S2

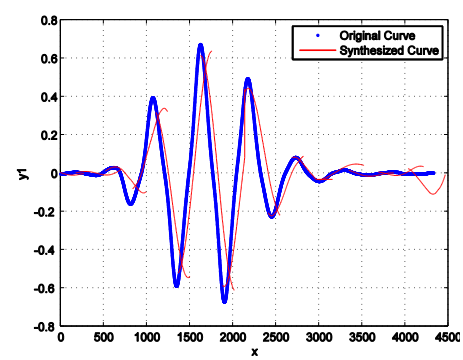

Figure 5: A plot of the synthesized template on the original, Normal S2, providing a good fit $\left(\mathrm{R}^{2}>0.9\right)$

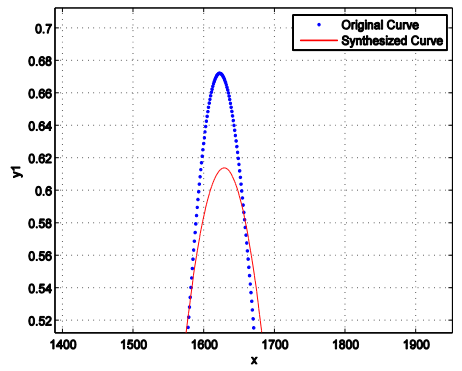

Figure 6: A magnified view of one of the peaks from Fig. 3, highlighting the fit of the synthesized complex to the original curve

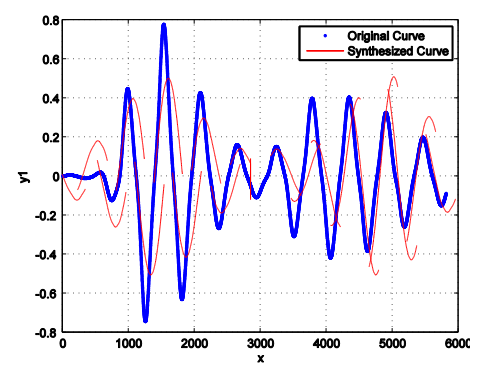

Figure 7: A plot of the template matching operation with a split S2 complex, giving a very poor fit $\left(\mathrm{R}^{2}<0.6\right)$

complex, with an $\mathrm{R}^{2}$ value of over 0.98 . The magnified view of the template matching operation with a normal S2 is shown in figure 6 . The template matching operation gives an $\mathrm{R}^{2}$ value of below 0.60 for complexes with splitting of S2 sounds as the fit is not good. This is clearly observed in figure 7 and in table 2 .

Table 2: A table of the R2 values obtained during the fitting of normal S2 template to original PCG signals with split S2

\begin{tabular}{|c|c|c|c|c|c|}
\hline Complex-1 & Complex-2 & Complex-3 & Complex-4 & Complex-5 & Complex-6 \\
\hline 0.5915 & 0.5798 & 0.5764 & 0.5796 & 0.5794 & 0.5733 \\
\hline Complex-7 & Complex-8 & Complex-9 & Complex-10 & Complex-11 & Complex-12 \\
\hline 0.5749 & 0.5816 & 0.4863 & 0.5803 & 0.5444 & 0.5260 \\
\hline
\end{tabular}

\section{Conclusion}

The previous section makes it clear that this approach is quite efficient in detecting split S2 complexes and the value of $\mathrm{R}^{2}$ can clearly indicate the presence of S2 splitting. The dependence of this method on a singular goodness of fit coefficient, to differentiate between normal and split S2 complexes, makes this method easier and faster to implement on digital systems. Only the basic operation of comparing the template coefficients to the data points on the original curve is required to implement this system, which can be done very easily on a FPGA board. Our method took a maximum of 0.6856 seconds to execute and generate results on an Intel core i3 processor with a 4GB RAM. Our future work will include the performance analysis of this method to already existing algorithms and its implementation on a FPGA board. 


\section{Books:}

\section{References}

[1] Howard B. Sprague, M.D., Patrick A. Ongley, M.D., "The Clinical Value of Phonocardiography”, Circulation. 1954;9:127-134

[2] Sandra LachArlinghaus, PHB Practical Handbook of Curve Fitting. CRC Press, 1994.

[3] William M. Kolb. Curve Fitting for Programmable Calculators. Syntec, Incorporated, 1984

\section{Journals:}

[4] Brooks, E.B.; Thomas, V.A; Wynne, R.H.; Coulston, J.W., "Fitting the Multitemporal Curve: A Fourier Series Approach to the Missing Data Problem in Remote Sensing Analysis," Geoscience and Remote Sensing, IEEE Transactions on , vol.50, no.9, pp.3340,3353, Sept. 2012,doi: 10.1109/TGRS.2012.2183137

[5] Brooks, E.B.; Thomas, V.A; Wynne, R.H.; Coulston, J.W., "Fitting the Multitemporal Curve: A Fourier Series Approach to the Missing Data Problem in Remote Sensing Analysis," Geoscience and Remote Sensing, IEEE Transactions on , vol.50, no.9, pp.3340,3353, Sept. 2012,doi: 10.1109/TGRS.2012.218313

[6] M. GULSEN, A. E. SMITH, D. M. TATE,"A genetic algorithm approach to curve fitting", International Journal of Production Research Vol. 33, Iss. 7, 1995

[7] 18. Songxin Liang, David J. Jeffrey, "An analytical approach for solving nonlinear boundary value problems in finite domains", Numerical Algorithms, Volume 56, Issue 1, pp 93-106, Springer US 2011, doi: 10.1007/s11075-010-9375-Z

[8] 19. Crockford DJ1, Keun HC, Smith LM, Holmes E, Nicholson JK. "Curve-fitting method for direct quantitation of compounds in complex biological mixtures using 1H NMR: application in metabonomic toxicology studies", Anal Chem. 2005 Jul 15;77(14):4556-62.PMID: 16013873

[9] 20. Regine Schmidt, Dirk Graafen, Stefan Weber, and Laura M. Schreiber. "Computational Fluid Dynamics Simulations of Contrast Agent Bolus Dispersion in a Coronary Bifurcation: Impact on MRI-Based Quantification of Myocardial Perfusion", Computational and Mathematical Methods in Medicine Volume 2013 (2013), Article ID 513187,http://dx.doi.org/10.1155/2013/513187

\section{Online Resources:}

[10] http://www.easyauscultation.com/heart-sounds

[11] Dugdale David C, Chen Michael A., Zieve D, "Heart murmur and sounds, Medline Plus, http://www.nlm.nih.gov/medlineplus/ency/article/003266 .htm"

[12] University of Michigan, Heart Sound and Murmur Library, http://www.med.umich.edu/lrc/psb/heartsounds/. 